\title{
EFFECTS OF SUPERVISED VS NON-SUPERVISED COMBINED AEROBIC AND RESISTANCE EXERCISE PROGRAMME ON CARDIOMETABOLIC RISK FACTORS
}

\author{
Tsvetan Stefanov', Anna Vekova1', Iveta Bonova', Stanislav Tzvetkov', Dimo Kurktschiev", Matthias Blüher², \\ Theodora Temelkova-Kurktschiev ${ }^{1}$ \\ ${ }^{1}$ Medicobiological Unit, International Scientific Institute, National Sports Academy, Sofia, Bulgaria \\ ${ }^{2}$ Department of Medicine, University of Leipzig, Leipzig, Germany
}

\section{SUMMARY}

Objective: We examined the effect of a 6-month combined aerobic and resistance training programme on cardiometabolic risk factors in nondiabetic subjects and compared its effectiveness when executed under strict professional supervision or without direct supervision.

Methods: Eighty-five sedentary, non-diabetic subjects (27 men and 58 women), mean age $47.5 \pm 0.6$ years, mean body mass index (BMI, $33.8 \pm 0.6 \mathrm{~kg} / \mathrm{m}^{2}$ ) participated in a combined exercise programme assigned to supervised ( $\left.\mathrm{S}, \mathrm{n}=31\right)$, non-supervised ( $\left.\mathrm{NS}, \mathrm{n}=24\right)$ or control group $(C, n=30)$. Cardiometabolic risk parameters were assessed at baseline and after the 6 -month training.

Results: In both the $S$ and NS group there was a significant decrease in BMI $\left(-1.6 \pm 0.3, p<0.001\right.$ and $\left.-1.0 \pm 0.3 \mathrm{~kg} / \mathrm{m}^{2}, p=0.004\right)$, waist circumference $(-10.1 \pm 1.1 \mathrm{~cm}, p<0.001$ and $-7.8 \pm 0.8 \mathrm{~cm}, p<0.001)$, fat mass $(-1.8 \pm 0.4 \%, p<0.001$ and $-2.1 \pm 0.6 \%, p=0.003)$, and a significant increase in fat-free mass $(+1.7 \pm 0.4 \%, p<0.001$ and $+2.0 \pm 0.7 \%, p=0.008)$, and aerobic capacity $(+6.9 \pm 1.1, p<0.001$ and $+6.9 \pm 0.8 \mathrm{ml} / \mathrm{kg}$ per min, $p=0.008)$. Fasting glucose did not change in $S$ and NS, but increased in $C(p=0.048)$. In the $S$ group a significant decrease in fasting insulin $(p<0.001)$, homeostasis model assessment of insulin resistance $(p<0.001)$, highly sensitive $C$-reactive protein $(p=0.004)$, leucocytes count $(p=0.04)$, systolic high $(p<0.001)$ and diastolic $(p=0.009)$ blood pressure was found. Comparable significant decreases in total and low-density lipoprotein cholesterol were observed in all study groups.

Conclusions: A 6-month combined exercise programme led to substantial improvement of various cardiometabolic risk factors. This programme was effective even when executed without direct supervision, although the effects were more pronounced in the supervised group. Our findings suggest that non-supervised exercise programmes may be a valuable, cost-effective tool to translate the current physical activity guidelines in a real-life setting.

Key words: overweight, obesity, metabolic disease, cardiovascular disease, physical activity

Address for correspondence: T. Stefanov, Medicobiological Unit, International Scientific Institute, National Sports Academy, 1700 Studentski Grad, Sofia, Bulgaria. E-mail: tsvetan.stefanov@gmail.com

\section{INTRODUCTION}

The prevalence of obesity has dramatically increased in the last few decades due to rapid changes in lifestyle and their interaction with genetic factors. Epidemiological studies indicate further growing tendency for the future (1-4). Obesity is associated with numerous metabolic derangements and is among the major causes for the development of insulin resistance, metabolic syndrome, type 2 diabetes mellitus (T2DM), and cardiovascular disease (5-7).

Sedentary lifestyle is a pathogenic factor of obesity development and obesity-related cardiometabolic complications (8-15). Increased physical activity and exercise training are well-established strategies for the treatment and prevention of obesity and its related diseases (16-19). Therefore, public health organizations recommend engagement in at least $150 \mathrm{~min}$ of moderate intensity aerobic physical activity (PA) in combination with 2 strength training sessions per week for all adults $(20,21)$. These recommendations are, however, based predominantly on evidence from studies evaluating the effect of both exercise modalities independently. To date, few trials have tested the impact of a combined aerobic and resistance training on cardiometabolic risk factors among overweight and obese individuals without history of T2DM (22-24).

Large-scale lifestyle and physical activity interventions were found to be effective in the treatment and prevention of obesity, metabolic syndrome, and T2DM among high-risk populations (25-29). These interventions have included regular counselling and high level of professional exercise supervision, thus being expensive and hard to implement on a population level. Recently, several studies demonstrated that the expensive lifestyle modifications and/or physical activity programmes could be successfully translated into a "real-world" setting. With this respect, the low-resource interventions used in the Ballarat Diabetes Prevention Pilot Initiative (BDPPI) (30), the Good Aging in Lahti Region (GOAL) (31), the Greater Green Triangle Study 
(GGTS) (32), and the Diabetes Education \& Prevention with a Lifestyle Intervention offered in the YMCA (DEPLOY) pilot study (33) substantially reduced diabetes risk and even 10-year coronary heart disease risk (34) among individuals predisposed to develop T2DM. Considering the rapidly growing prevalence of physical inactivity and the associated health care burden, more easily affordable exercise strategies are also needed. Programmes comprising an initial supervised exercise course and provision of regular exercise advice thereafter might represent a valuable, cost-effective tool to translate the physical activity guidelines on community level.

Thus, in the present study we aimed to: 1) test the effect of a 6-month training programme combining aerobic and resistance type exercises in accordance with the current physical activity guidelines on cardiometabolic risk factors and 2) compare the effectiveness of a supervised vs. non-supervised combined exercise programme among sedentary, middle-aged, overweight/obese, non-diabetic individuals.

\section{MATERIALS AND METHODS}

\section{Study Design and Subjects}

Combating the Metabolic Syndrome through Physical Activity (COMPACT) is a 6-month study designed to: 1 ) test the feasibility of an exercise programme combining aerobic and resistance type exercises in accordance with the current PA guidelines and its effect on various cardiometabolic risk factors, 2) compare the effectiveness of a supervised vs. non-supervised combined exercise programme in accordance with the current PA guidelines among sedentary middle-aged, overweight and obese individuals without T2DM. The COMPACT study was conducted at the International Scientific Institute, National Sports Academy, Sofia, Bulgaria (Fig. 1).

Through postings at the University campus and announcements on the web page of the University, on the Internet, and in the local newspapers, 141 individuals admitted to initial interview were $40-60$ years old, had body mass index (BMI) $\geq 25 \mathrm{~kg} / \mathrm{m}^{2}$ and were physically inactive at the time of enrolment (a lack of engagement in structured PA of moderate to high intensity for more than 30 min 2 times per week).

Involvement in the study was not permitted if any of the following exclusion criteria was met: medical history of T2DM; presence of a cardiovascular disorder or abnormality: arrhythmias, congenital heart defects, ischaemic heart disease; myocardial infarction or stroke in the last 6 months; poorly controlled hypertension ( $\geq 160 / 110 \mathrm{mmHg}$ ); presence of neurological, thyroid, hepatic, renal or musculoskeletal disease; presence of acute or chronic inflammatory disease; adherence to a weight-loss diet or body weight change of more than $\pm 3 \mathrm{~kg}$ in the last 3 months; use of medications or nutritional supplements that may affect energy expenditure and body weight, lipid and carbohydrate metabolism; drug or alcohol abuse. Based on these criteria 94 subjects out of the initially interviewed 141 were included in the baseline examination. Of them, 9 individuals turned out to have a so far undiagnosed T2DM with fasting blood glucose $\geq 7.0 \mathrm{mmol} / 1$ and were excluded from further participation. The remaining 85 subjects were enrolled in three study groups matched for age, sex and BMI: the supervised PA group $(\mathrm{S}, \mathrm{n}=31)$, the non-supervised PA group (NS, $n=24)$, or the control group $(C, n=30)$. Two subjects dropped out from S and NS, respectively, and two subjects from the $\mathrm{C}$ group were lost to follow-up. Thus, 29 subjects from S, 22 from NS, and 28 from the $\mathrm{C}$ group completed the study and were included in the final analyses (Fig. 1).

The study conforms to the principles outlined in the Declaration of Helsinki. All subjects were provided with a detailed explanation of the study aims and gave their written informed consent prior to the baseline medical examination.

\section{Exercise Programme}

All participants were provided healthy lifestyle counselling emphasizing the health benefits of regular PA and balanced nutrition at a specially designed lecture at the start of the study. In addition, every subject had a scheduled 1-hour personal meeting with a dietician at which he/she was given individually tailored advice and was provided with a standardized low calorie diet (1,400 $\mathrm{kcal}$ and $1,800 \mathrm{kcal}$ per day, for women and men, respectively) consisting of $55 \%$ of total energy intake from carbohydrates, $30 \%$ from fat, and $15 \%$ from protein. Thereafter, subjects allocated to the $\mathrm{C}$ group were not contacted for the next 6 months until the final study visit.

Subjects in the S and NS physical activity group participated in an intensive 6-month physical exercise programme in accordance with the current PA guidelines promoting engagement in at least $150 \mathrm{~min}$ of aerobic exercises in combination with 2 sessions of resistance exercises per week.

All training sessions incorporated endurance exercises (e.g. brisk walking, low-intensity running, aerobics, tae-bo, dancing), resistance exercise (e.g. free weight exercises, own body weight exercises, resistance bands exercises, abdominal crunches), and flexibility exercises (e.g. stretching of the major muscle groups).

Since subjects were physically inactive prior to enrolment, they participated in a 1-week exercise course consisting of 2 lectures and 3 practical sessions during which they got acquainted with the basic principles of sports training and the execution of different exercises. Subsequently, during the initial 10 weeks of the study, participants were encouraged to perform 2 exercise sessions per week with low to moderate intensity (aerobic exercises at $50-60 \%$ of the maximum heart rate; resistance exercise for the major muscle groups in 2 sets of 8-14 repetitions) and duration of 30-35 min. For 10 consecutive weeks, the number of sessions was raised to $3 /$ week and their intensity (aerobic exercises at $60-75 \%$ of maximum heart rate; resistance exercises in 3 sets of 8-14 repetitions) and duration (45-60 $\mathrm{min}$ ) was also increased. During the last 4 weeks of the study the goal was participation in 4 training sessions per week (45-60 min). The overall aim was participation in 66 exercise sessions for the whole study duration.

The supervised and the non-supervised programmes differed with respect to PA advice regularity and degree of professional supervision. In the $\mathrm{S}$ group all training sessions were conducted and supervised by a trained physical therapist. Subjects in the NS group were encouraged to achieve the study aim without direct supervision. However, personal meetings with a physical therapist were scheduled once in 3 weeks in order to evaluate the progress and set up new activity goals. 
Exercise participation was verified by direct supervision in the supervised group and by exercise diary and telephone interviews in the non-supervised group.

\section{Measurements}

All measurements were performed at baseline and after the 6-month exercise programme between 8:00 a.m. and 10:00 a.m. after a 10-hour overnight fast. Subjects were asked to refrain from strenuous exercise as well as from food excess or deprivation 3 days prior to both study visits.

\section{Anthropometry and Blood Pressure Assessment}

Anthropometric measurements were performed according to standard techniques with subjects wearing light clothing. Blood pressure was examined in a sitting position after a rest of at least 5 minutes. Two consecutive measurements were performed within 3 minutes and the second one was taken into consideration.

\section{Body Composition Analysis}

Body composition analysis was performed using the bio impedance technique on a TANITA BC 418 MA analyser (TANITA Corporation, Tokyo, Japan).

\section{Sub-maximal Aerobic Capacity Assessment}

Sub-maximal aerobic capacity was assessed on a treadmill (Quasar Med 4.0, HP Cosmos, Nussdorf-Traunstein, Germany) using the Modified Bruce protocol (35). Maximal aerobic capacity $\left(\mathrm{VO}_{2} \max \right)$ was then estimated using the equation of Foster (36).

\section{Other Measurements}

At baseline and after the 6-month exercise programme medical history was taken and physical examination was performed. In addition, participants completed the short version of the International Physical Activity Questionnaire (IPAQ) and a questionnaire concerning their motivation for participation in the study as well as their self-perception of current psychological and health status. At baseline, 12-lead resting ECG was performed in order to exclude the presence of any acquired or congenital heart disease. At the end of the study subjects underwent a detailed interview concerning the factors that have stimulated or limited their participation in the exercise programme.

\section{Blood Sample Analyses}

Venous blood samples were obtained by venipuncture. EDTA plasma and serum were separated by centrifugation (4000 rpm for 8 minutes at $4^{\circ} \mathrm{C}$ ). Aliquots of plasma and serum were immediately stored at $-40^{\circ} \mathrm{C}$ until further analyses. Fasting glucose, lipid profile, and leucocytes count were determined using fresh material.

Leucocytes count was determined photometrically on a Diagon D5 Cell analyser (Diagon Ltd., Budapest, Hungary). Fasting glucose was measured in serum by the glucose-hexokinase method on an Integra 400 Plus analyser (Roche Diagnostics GmbH, Mannheim, Germany). Fasting serum total cholesterol, high-density lipoprotein (HDL) cholesterol, and triglyceride concentrations were measured enzymatically on an Integra 400 Plus analyser (Roche Diagnostics $\mathrm{GmbH}$ ). Serum low-density lipoprotein (LDL) cholesterol concentration was calculated using
Friedewald equation. Fasting insulin concentration was measured in serum by ELISA (Mecordia, Uppsala, Sweden; inter-assay variability: $2.9 \%$; intra-assay variability: $3.4 \%$ ), which shows no cross-reactivity with proinsulin. Insulin resistance was calculated from fasting serum glucose and fasting serum insulin using the homeostasis model assessment for insulin resistance (HOMA-IR). Serum high sensitive C-reactive protein (hs-CRP) concentration was measured by ELISA (ap Dianv, Turnhout, Belgium; interassay variability: $6.1 \%$; intra-assay variability: $5.1 \%$ ).

\section{Statistical Methods}

Data are presented as n, mean \pm SEM, or percentage (\%) as respectively indicated. The distribution of variables was assessed by the Kolmogorov-Smirnov test for homogeneity of variance and non-normally distributed variables were logarithmically transformed to approximate normal distribution. One-way ANOVA was used for between-group comparisons at baseline and at the end of the intervention. Within-group changes were analysed using paired sample Student's t test. Multiple linear regression analyses were performed to determine the predictors of changes in BMI, waist circumference and insulin resistance (HOMA-IR). Differences were considered statistically significant at $\mathrm{P}<0.05$. All statistical analyses were executed using SPSS for Windows, version 17.0 (SPSS Inc., Chicago, IL).

\section{RESULTS}

Baseline demographic, anthropometric, and metabolic characteristics of the study participants are presented in Table 1. No significant between-group differences were observed except for LDL-cholesterol, which was significantly higher in the nonsupervised vs. the supervised PA group $(p=0.02)$.

As a result of the 6-month combined exercise programme body weight and BMI decreased significantly in the $S(p<0.001$ for both) and the NS group ( $\mathrm{p}=0.005$ and $\mathrm{p}=0.004$, respectively), and slightly increased in the $\mathrm{C}$ group ( $\mathrm{p}>0.05$ for both) (Fig. $2 \mathrm{a}, 2 \mathrm{~b}$ and $2 c)$. Waist circumference degreased significantly from baseline in all three study groups (Fig. 2d). Statistically significant favourable changes in fat mass and fat-free mass were found in the $S(p<0.001$ for both) and the NS group ( $\mathrm{p}=0.003$ and $\mathrm{p}=0.008$, respectively), whereas in the $\mathrm{C}$ group a tendency towards an increase in fat mass and a decrease in fat-free mass was present (Fig. 2e and 2f). The magnitude of changes in body weight, BMI, waist circumference, and body composition was comparable between the S and the NS group ( $p>0.05$ for all variables), and was significantly greater in both PA groups when compared to the group $\mathrm{C}(\mathrm{p}<0.01$ for all variables) (Fig. 2).

After the exercise programme a comparable increase in maximal aerobic capacity $\left(\mathrm{VO}_{2} \mathrm{max}\right)$ was observed within the $\mathrm{S}$ and the NS group ( $<<0.001$ for the change in both groups), while it remained unchanged in the group $\mathrm{C}(\mathrm{p}>0.05)$ (Fig. 3).

Changes in lipid profile, glucose homeostasis, inflammation, and blood pressure (BP) as a result of the combined exercise programme are depicted in Table 2. Total and LDL cholesterol concentrations decreased significantly from baseline in all three groups, while HDL cholesterol and triglyceride levels remained unchanged. Fasting glucose concentration increased within the 
Table 1: Baseline characteristics of study participants

\begin{tabular}{|c|c|c|c|}
\hline Characteristic & Supervised group & Non-supervised group & Control group \\
\hline $\mathrm{n}$ (men/women) & $29(10 / 19)$ & $22(8 / 14)$ & $28(9 / 19)$ \\
\hline Age (years) & $47.8 \pm 1$ & $47.8 \pm 1.3$ & $46.8 \pm 1.2$ \\
\hline Body weight (kg) & $94.7 \pm 4.8$ & $93.7 \pm 4.2$ & $98.7 \pm 3$ \\
\hline Body mass index $\left(\mathrm{kg} / \mathrm{m}^{2}\right)$ & $33.7 \pm 1.2$ & $33.4 \pm 1.4$ & $34.0 \pm 1$ \\
\hline Waist circumference $(\mathrm{cm})$ & $107.4 \pm 3$ & $104.6 \pm 2.9$ & $107.7 \pm 2.3$ \\
\hline Fat mass $(\%)$ & $38.5 \pm 6.8$ & $38.9 \pm 1.7$ & $38.8 \pm 1.4$ \\
\hline Fat-free mass (\%) & $61.3 \pm 1.3$ & $61.0 \pm 1.7$ & $61.0 \pm 1.4$ \\
\hline $\mathrm{VO}_{2} \max (\mathrm{ml} / \mathrm{kg}$ per min) & $32.1 \pm 2.4$ & $32.5 \pm 2.9$ & $33.5 \pm 2.1$ \\
\hline Total cholesterol (mmol/l) & $5.50 \pm 0.2$ & $6.14 \pm 0.2$ & $5.97 \pm 0.2$ \\
\hline LDL cholesterol (mmol/l) & $3.33 \pm 0.2^{*}$ & $3.95 \pm 0.2$ & $3.73 \pm 0.1$ \\
\hline HDL cholesterol (mmol/l) & $1.47 \pm 0.1$ & $1.46 \pm 0.1$ & $1.42 \pm 0.1$ \\
\hline Triglycerides (mmol/l) & $1.54 \pm 0.2$ & $1.60 \pm 0.2$ & $1.63 \pm 0.2$ \\
\hline Fasting glucose (mmol/l) & $5.66 \pm 0.1$ & $5.41 \pm 0.1$ & $5.44 \pm 0.1$ \\
\hline Fasting insulin (pmol/l) & $11.35 \pm 1.1$ & $9.06 \pm 1.3$ & $11.04 \pm 1.5$ \\
\hline HOMA-IR & $2.88 \pm 0.3$ & $2.23 \pm 0.4$ & $2.77 \pm 0.5$ \\
\hline Systolic blood pressure $(\mathrm{mmHg})$ & $136 \pm 3.1$ & $126 \pm 3.3$ & $132 \pm 3.1$ \\
\hline Diastolic blood pressure (mmHg) & $89 \pm 1.6$ & $86 \pm 2.1$ & $86 \pm 1.5$ \\
\hline hs-CRP (mg/l) & $3.3 \pm 0.4$ & $3.2 \pm 0.6$ & $3.3 \pm 0.6$ \\
\hline Leucocytes count (GPt/l) & $8.6 \pm 0.5$ & $7.6 \pm 0.4$ & $8.2 \pm 0.4$ \\
\hline
\end{tabular}

Data are presented as $n$, mean $\pm S E M$, or $\%$ as respectively indicated

${ }^{*} p=0.02$ vs. non-supervised group

Table 2: Changes in various cardiometabolic risk factors as a result of the exercise programme

\begin{tabular}{|c|c|c|c|}
\hline Characteristic & Supervised group & Non-supervised group & Control group \\
\hline Total cholesterol (mmol/l) & $-0.32 \pm 0.1^{*}$ & $-0.58 \pm 0.2^{\star}$ & $-0.35 \pm 0.1^{*}$ \\
\hline LDL cholesterol (mmol/l) & $-0.38 \pm 0.1^{*}$ & $-0.57 \pm 0.12^{*}$ & $-0.28 \pm 0.1^{*}$ \\
\hline HDL cholesterol (mmol/l) & $0.03 \pm 0.05$ & $0.003 \pm 0.1$ & $-0.01 \pm 0.04$ \\
\hline Triglycerides (mmol/l) & $0.05 \pm 0.1$ & $-0.01 \pm 0.2$ & $-0.04 \pm 0.1$ \\
\hline Fasting glucose $(\mathrm{mmol} / \mathrm{l})$ & $-0.16 \pm 0.1$ & $0.01 \pm 0.2$ & $0.15 \pm 0.1^{*}$ \\
\hline Fasting insulin (pmol//) & $-3.24 \pm 0.9^{*} \ddagger$ & $-0.14 \pm 0.9$ & $-1.46 \pm 1.1$ \\
\hline HOMA-IR & $-0.88 \pm 0.2^{*} \ddagger$ & $-0.01 \pm 0.3$ & $-0.36 \pm 0.3$ \\
\hline Systolic blood pressure (mmHg) & $-10.7 \pm 2.4^{*} \dagger \ddagger$ & $-1.8 \pm 2.9$ & $-2.3 \pm 1.8$ \\
\hline Diastolic blood pressure $(\mathrm{mmHg})$ & $-4.8 \pm 1.7^{*} \dagger$ & $-2.3 \pm 1.9$ & $0.9 \pm 1.1$ \\
\hline hs-CRP (mg/l) & $-0.92 \pm 0.3^{*} \dagger$ & $-0.26 \pm 0.3$ & $-0.05 \pm 0.3$ \\
\hline Leucocytes count (GPt/l) & $-0.7 \pm 0.3^{*}$ & $-0.4 \pm 0.3$ & $-0.4 \pm 0.2$ \\
\hline
\end{tabular}

Data are presented as mean \pm SEM, or $\%$ as respectively indicated

${ }^{*} p<0.05$ for within group change from baseline to final visit

$\dagger p<0.05$ vs. control group

$\ddagger p<0.05$ vs. non-supervised group

group $C(p=0.048)$ and remained unchanged in both exercise groups. Fasting insulin and HOMA-IR decreased significantly from baseline only in the group $\mathrm{S}$ ( $\mathrm{p}=0.001$ for both). Similarly, a significant decrease from baseline in leucocytes count $(\mathrm{p}=0.04)$, hs-CRP $(p=0.004)$ as well as in systolic $(p<0.001)$ and diastolic $(\mathrm{p}=0.009) \mathrm{BP}$ was observed only in the group S (Table 2).

Subjects in the supervised group attended $73.4 \pm 6.7 \%$ of all exercise sessions or $2 \pm 0.2$ sessions per week, whereas those in the non-supervised group completed $54.8 \pm 5.6 \%$ of the prescribed exercise sessions or 1.5 sessions per week. Thus, adherence to the prescribed exercise was significantly greater in the supervised vs. the non-supervised group $(\mathrm{p}=0.047)$.

Multiple linear regression analyses revealed the number of attended training sessions as an independent determinant of change in BMI, change in waist circumference, and change in HOMA-IR (Table 3).

No differences in the effect of the exercise programme on the investigated cardiometabolic risk factors were found between men and women. Similarly, no gender differences in exercise adherence were present (data not shown). 
Table 3: Multiple linear regression analyses of changes in various cardiometabolic risk factors and the number of attended training sessions as determinants of changes $(\Delta)$ in BMI, waist circumference, and HOMA-IR

\begin{tabular}{|c|c|c|}
\hline \multirow{3}{*}{ Determinants } & \multicolumn{2}{|c|}{ Dependent variables } \\
\hline & \multicolumn{2}{|c|}{$\Delta \mathrm{BMI}$} \\
\hline & $\beta$ coefficient & p-value \\
\hline Age & 0.052 & ns \\
\hline Sex & 0.111 & ns \\
\hline BMl at baseline & 0.298 & 0.02 \\
\hline \multirow[t]{2}{*}{ Number of training sessions } & 0.524 & $<0.001$ \\
\hline & \multicolumn{2}{|c|}{$\Delta$ waist circumference } \\
\hline Age & 0.252 & ns \\
\hline Sex & 0.107 & ns \\
\hline Waist circumference at baseline & 0.030 & ns \\
\hline \multirow[t]{2}{*}{ Number of training sessions } & 0.647 & $<0.001$ \\
\hline & \multicolumn{2}{|c|}{$\triangle$ HOMA-IR } \\
\hline Age & 0.115 & ns \\
\hline Sex & 0.018 & ns \\
\hline$\Delta \mathrm{BMI}$ & -0.170 & ns \\
\hline$\Delta$ waist circumference & 0.160 & ns \\
\hline Number of training sessions & 0.335 & 0.034 \\
\hline HOMA-IR at baseline & 0.604 & $<0.001$ \\
\hline$\Delta$ leucocytes count & -0.154 & ns \\
\hline$\Delta$ hs-CRP & 0.008 & ns \\
\hline
\end{tabular}

ns - not significant

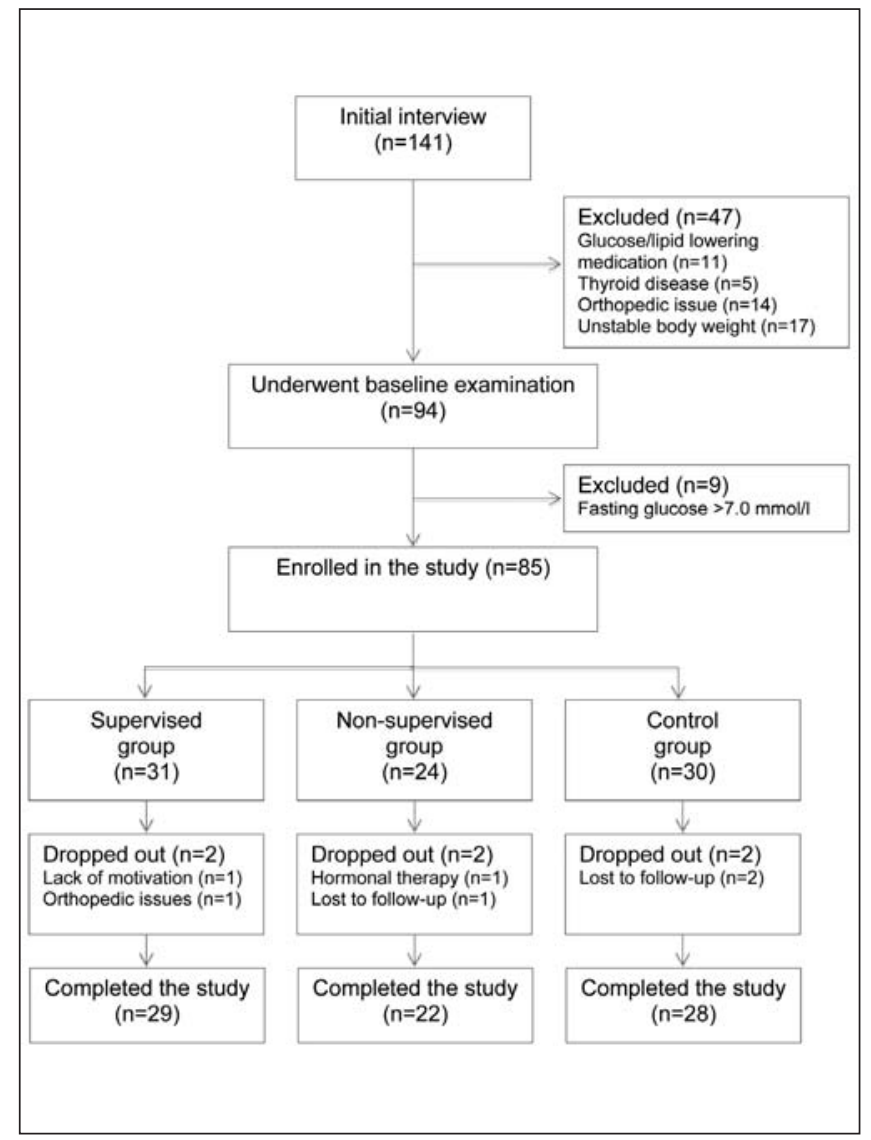

Fig. 1. Study flow chart.

\section{DISCUSSION}

The main findings of the COMPACT study were that: 1) training programme combining aerobic and resistance type exercises in accordance with the current PA guidelines led to substantial improvements in various cardiometabolic risk factors among previously inactive, overweight/obese, middle-aged individuals without T2DM and 2) this programme was effective also without direct supervision, although the effect was more strongly expressed when executed under strict professional supervision. These findings confirm and further extend earlier reports on the health effects of combined aerobic and resistance exercise training and suggest that programmes comprising an initial supervised exercise course and provision of regular exercise advice thereafter may be a valuable, cost-effective tool to translate the current PA guidelines in a real-life setting.

In the current work, a significant decrease in body weight, BMI, and waist circumference and substantial beneficial changes in body composition occurred as a result of the exercise training. This is in agreement with some but not all of the reports on the effect of combined exercise on anthropometric risk factors in overweight/obese individuals. Thus, Stensvold et al. (24) observed a decrease in waist girth and no changes in body weight and BMI after 12 weeks of combined exercise training. Recently, Bateman et al. (22) and Slentz et al. (23) found significant changes in body mass and waist circumference after 8 months of training in obese individuals. In contrast, however, others did not find an effect of combined training on these anthropometric variables (37). Data 


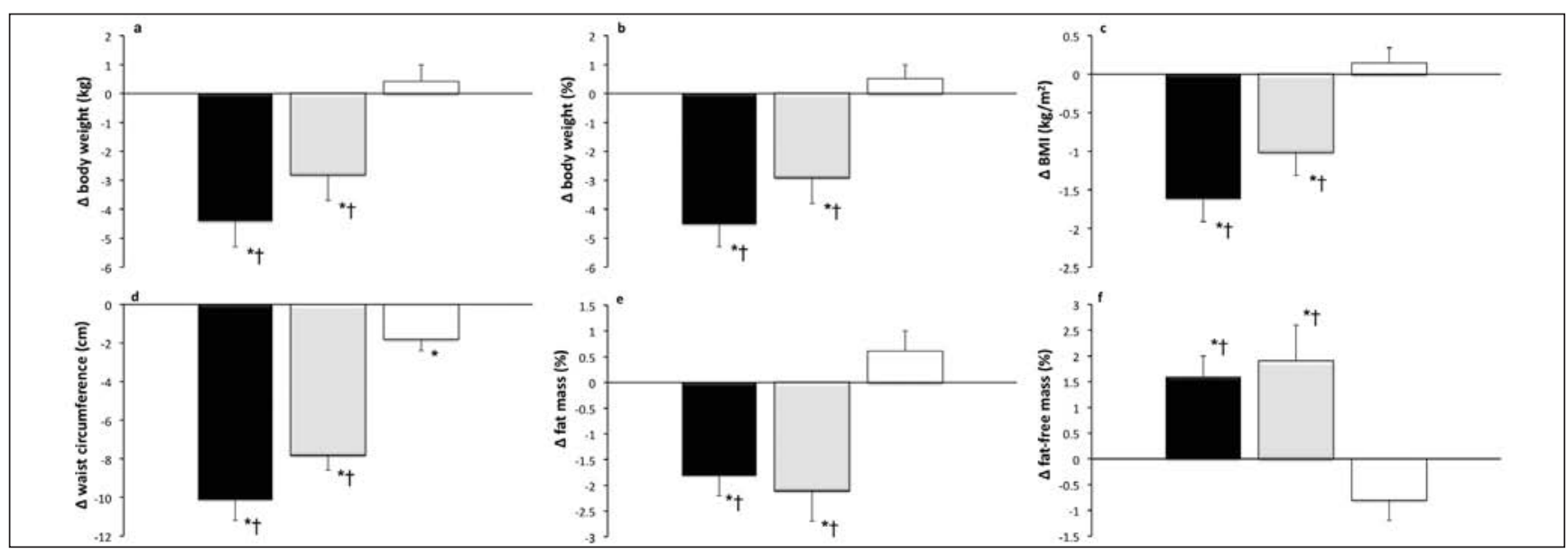

Fig. 2. Representing a) absolute change in body weight, b) \% change in body weight, c) change in body mass index, d) change in waist circumference, e) change in \% fat mass, and f) change in \% fat-free mass as a result of the combined exercise programme; black bars: supervised group; grey bars: non-supervised group; white bars: control group.

${ }^{*} p<0.05$ for within group change from baseline to final visit; $\uparrow p<0.05$ vs. control group; values are mean $\pm S E M$

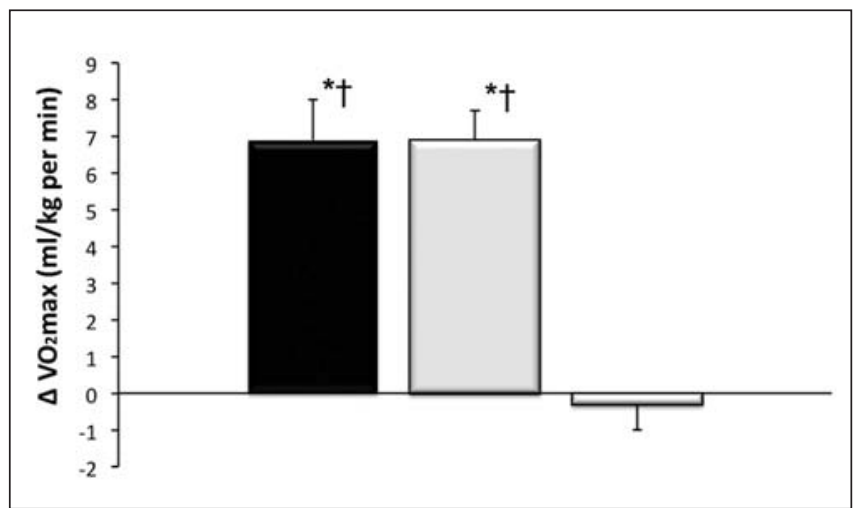

Fig. 3. Changes in maximal aerobic capacity ( $\left.\mathrm{VO}_{2} \mathrm{max}\right)$ as a result of the combined exercise programme; black bars: supervised group; grey bars: non-supervised group; white bars: control group.

${ }^{*} p<0.05$ for within group change from baseline to final visit; $\uparrow p<0.05$ vs. control group; values are mean \pm SEM

concerning the impact of combined training on body composition are more consistent. In line with our findings, a decrease in fat mass and an increase in lean body mass after 12 weeks and 8 months of exercise, respectively, were previously reported $(23,24)$.

The changes in body weight, waist circumference, and body composition found in the present study were comparable between the $\mathrm{S}$ and the NS group, which corroborates previous observations $(30-33,38)$. Perri et al. (38) for example reported equal decrease in body weight as a result of a group- and a home-based exercise programme in obese women. In addition, Payne et al. (30) found comparable changes in body mass and waist circumference after a gymnasium- and a home-based resistance exercise programme in the BDPPI. Similar findings were observed in response to the low-resource lifestyle and PA interventions used in the GOAL (31), GGTS (32), and the DEPLOY study (33).

Poor cardiorespiratory fitness is an independent predictor of metabolic syndrome, T2DM, and cardiovascular disease, while high aerobic capacity is associated with lower cardiometabolic disease prevalence (39-41). Substantial improvements in maximal aerobic capacity were previously observed among middle-aged, overweight or obese individuals after 12 (24), 16 weeks (37) and 8 months $(22,23)$ of combined exercise, respectively. Accordingly, we found a significant increase in $\mathrm{VO}_{2}$ max after 6 months of training. The increase in aerobic capacity was equivalent in both physical activity groups, which is in agreement with the observations of others, who reported comparable increases in $\mathrm{VO}_{2}$ max after group- and home-based exercise interventions $(38,42)$. This finding is, however, surprising since the exercise adherence was higher in the supervised group and one might expect significantly higher increase in $\mathrm{VO}_{2} \mathrm{max}$ in this group compared to the nonsupervised. The discrepancy may be explained by different factors such as higher adherence to aerobic exercise despite lower total exercise adherence in the NS group. On the other hand, in the present study aerobic capacity was estimated after performance of sub-maximal aerobic capacity test and not directly measured by spiroergometry. Thus, the method used for aerobic capacity assessment might have not been sensitive enough to detect small changes in $\mathrm{VO}_{2} \max$.

As a result of the 6-month combined training significant decreases in total and LDL cholesterol concentrations were observed, but HDL cholesterol and triglyceride levels remained unchanged. In general, physical exercise is associated with beneficial changes in lipid profile, although controversial findings have been reported (43). Data on the influence of combined training on blood lipids is also ambiguous. Thus, Libardi et al. (37) and Bateman et al. (22) found significant decrease in total cholesterol (37) and triglyceride concentration $(22,37)$ after 16 weeks and 8 months of combined training, respectively, in overweight and obese individuals. In contrast, however, Stensvold et al. (24) did not observe an effect on these parameters after 12 weeks of exercise. In none of these studies an effect of combined exercise on LDL and HDL cholesterol was reported.

In the present study, the $\mathrm{S}$ and the NS exercise groups exhibited comparable changes in lipid profile. Accordingly, significant improvements in total and LDL cholesterol have been observed after home-based and cost-effective community-based interventions 
$(30,32,33)$. In some of these trials beneficial changes in HDL cholesterol and/or triglycerides have also been present, potentially due to the frequent dietary counselling and the emphasis on dietary modification $(30,32)$.

No changes in fasting glucose concentration occurred as a result of the 6-month exercise programme in the present study, which is in line with previous reports on the effect of combined exercise among overweight or obese individuals $(22,24,37)$. Recently, Payne et al. (30) reported comparable significant changes in fasting glucose levels after a gymnasium- and a home-based resistance training programme. Similar findings were also reported in the low-resource GOAL study and GGTS $(31,32)$. In all these trials, however, subjects were selected to have impaired glucose tolerance and other T2DM risk factors. The COMPACT study participants were sedentary and overweight/obese, but had normal glucose levels at baseline, which may to a certain extent explain the discrepancy.

Noteworthy is the fact that while fasting glucose did not change in the S and in the NS group, a significant increase in glucose level was observed in the group $\mathrm{C}$. Based on this finding it is tempting to suggest that the exercise programme prevented a potential increase in fasting glycemia among subjects in the physical activity groups.

Strong relationship between PA and insulin sensitivity even among healthy individuals has been repeatedly reported $(44,45)$, but information on the effect of combined exercise on insulin sensitivity is sparse. In agreement with our findings, Slentz et al. (23) recently found significant improvement in insulin resistance (assessed by HOMA-IR) after 8 months on combined training in obese adults. On the contrary, Stensvold et al. (24) did not observe a change in HOMA-IR after 12 weeks of training among obese individuals most likely due to the relatively short duration of the study.

Furthermore, physical exercise has been found to improve insulin sensitivity through changes in body weight, but also through weight-loss independent mechanisms (46). Accordingly, in our study, the number of attended training sessions remained a significant predictor of the change in HOMA-IR after adjustment for changes in BMI (Table 3).

After 6 months of exercise training insulin resistance improved significantly in the $\mathrm{S}$ and remained unchanged in the NS group. Subjects in the NS group were somewhat more insulin sensitive at baseline, which may to a certain extent explain the difference. However, it is also very likely that the lack of effect in the NS group is due to lower adherence to exercise prescription, since multiple regression analysis revealed the number of attended training sessions as an independent determinant of the change in HOMA-IR.

In the present study, the combination of aerobic and resistance exercises led to a significant reduction in leucocytes count and hs-CRP concentration. PA has been suggested to decrease cardiometabolic disease risk by reducing subclinical inflammation (47), but reports on the effect of exercise on inflammatory biomarkers remain controversial $(48,49)$. Hitherto, only one study has tested the effect of combined training on inflammation among overweight subjects, with no effect on CRP concentration (37).

The positive effect of exercise on resting BP is well established $(50,51)$. Similarly to aerobic or resistance training alone, combined exercise was recently found to decrease BP (22).
In accordance with these findings, we also observed significant reduction in systolic and diastolic BP in response to exercise.

In the current work, significant changes in BP and markers of inflammation were only present in the S group. Similarly, Payne et al. (30) found a more pronounced decrease in systolic blood pressure in the supervised group of the BDPPI. A plausible explanation of the lack of effect on BP and the examined inflammatory biomarkers in the non-supervised group may be the lower exercise adherence.

At the end of the study, significant decreases in waist circumference as well as in total and LDL cholesterol concentrations were also observed in the control group. Subject in this group did not participate in a structured exercise programme, but received healthy lifestyle counselling at baseline and were provided a standard low-calorie diet. Thus, it may be assumed that some of the previously inactive and overweight/obese individuals adopted the provided advice, increased their leisure time PA and modified their dietary habits.

In order to determine whether there were any differences in the effect of the exercise programme on the investigated cardiometabolic risk factors as well as in exercise adherence, we conducted all the analyses separately among men and women. The results in both genders replicated those observed in the whole study population and no between-gender differences were found. Thus, it might be concluded that our results are not only valid for the whole study population in general, but also for both genders.

All subjects included in the present study were provided tailored dietary advice and were given a standard low calorie diet at baseline. During the study no additional dietary counselling was provided and neither the dietary adherence nor the change in dietary habits was evaluated. This may be considered a major limitation of the study, since the potential contribution of a dietary modification to the effect of the exercise on the investigated parameters cannot be distinguished. While providing standardized diets to the subjects in all 3 study groups, we aimed at experimental control for the effect of the diet on the exercise training outcomes. However, a hypothetical effect of the diet on the investigated cardiometabolic risk factors cannot be excluded. Nevertheless, dietary counselling is an integral part of every lifestyle modification programme and thus the design of our study reflects to a great extent a potential real-life situation in which a supervised or a non-supervised exercise programme may be used for metabolic disease prevention.

In summary, a 6-month training programme combining aerobic and resistance type exercises in accordance with the current PA guidelines led to substantial improvements in various cardiometabolic risk factors among previously sedentary, overweight or obese individuals, without T2DM. This programme was effective also in the group without direct supervision, although the effect was more strongly expressed when executed under strict professional supervision. Our findings indicate that combined exercise may improve cardiometabolic risk and suggest that low-resource programmes comprising baseline exercise course and regular PA advice thereafter, may represent an effective tool to translate the current PA guidelines into a "real-world" setting.

\section{Acknowledgements}

The authors would like to thank all study participants and Daniela Kox, Manuela Prellberg, and Susan Berthold for their technical assistance. 


\section{Conflict of Interest}

None declared

\section{Ethical Standards}

The authors of the current manuscript declare that all the experiments comply with the current laws of the country in which they were performed (Bulgaria). The study conforms to the principles outlined in the Declaration of Helsinki. The study protocol was approved by the Institutional Review Board of the National Sports Academy, Sofia, Bulgaria, and all subjects gave their written informed consent prior to participation.

\section{REFERENCES}

1. Temelkova-Kurktschiev T, Stefanov T. Lifestyle and genetics in obesity and type 2 diabetes. Exp Clin Endocrinol Diabetes. 2012 Jan;120(1):1-6.

2. Wang Y, Beydoun MA, Liang L, Caballero B, Kumanyika SK. Will all Americans become overweight or obese? estimating the progression and cost of the US obesity epidemic. Obesity (Silver Spring). 2008 Oct;16(10):2323-30.

3. Von Ruesten A, Steffen A, Floegel A, van der A DL, Masala G, Tjønneland A, et al. Trend in obesity prevalence in European adult cohort populations during follow-up since 1996 and their predictions to 2015. PLoS One. 2011;6(11):e27455

4. Stefanov T, Vekova A, Kurktschiev D, Temelkova-Kurktschiev T. Relationship of physical activity and eating behavior with obesity and type 2 diabetes mellitus: Sofia Lifestyle (SLS) study. Folia Med (Plovdiv). 2011 Jan-Mar;53(1):11-8

5. Van Gaal LF, Mertens IL, De Block CE. Mechanisms linking obesity with cardiovascular disease. Nature. 2006 Dec 14;444(7121):875-80

6. Stefanov TS, Temelkova-Kurktschiev TS. The metabolic syndrome in Bulgaria. Folia Med (Plovdiv). 2011 Oct-Dec;53(4):5-14.

7. Vangelova K, Deyanov C. Dyslipoproteinemias in industrial workers relationship with hypertension and overweight. Cent Eur J Public Health. 2000 May;8(2):77-9.

8. Hu FB. Sedentary lifestyle and risk of obesity and type 2 diabetes. Lipids. 2003 Feb;38(2):103-8.

9. Grøntved A, Hu FB. Television viewing and risk of type 2 diabetes, cardiovascular disease, and all-cause mortality: a meta-analysis. JAMA. 2011 Jun 15;305(23):2448-55

10. Stefanov T, Temelkova-Kurktschiev T, Koehler C, Henkel E, Schaper F, Hanefeld M. Association of physical activity with insulin resistance, subclinical inflammation, coagulation, and fibrinolytic biomarkers among population at high risk for type 2 diabetes. Folia Med (Plovdiv). 2012 Apr-Jun;54(2):32-9.

11. Kwaśniewska M, Kaleta D, Dziankowska-Zaborszczyk E, Drygas W. Healthy behaviours, lifestyle patterns and sociodemographic determinants of the metabolic syndrome. Cent Eur J Public Health. 2009 Mar; 17(1):14 9.

12. Pikala M, Kaleta D, Bielecki W, Maniecka-Bryła I, Drygas W, Kwaśniewska M. Awareness of cardiovascular prevention methods among residents of post-communist Polish provinces with highest mortality rates. Cent Eur J Public Health. 2011 Dec;19(4):183-9.

13. Vitáriušová E, Babinská K, Košt'álová L, Rosinský J, Hlavatá A, Pribilincová $\mathrm{Z}$, et al. Food intake, leisure time activities and the prevalence of obesity in schoolchildren in Slovakia. Cent Eur J Public Health. 2010 Dec;18(4):192-7.

14. Humeníková L, Gates GE. Dietary intakes, physical activity, and predictors of child obesity among 4-6th graders in the Czech Republic. Cent Eur J Public Health. 2007 Mar;15(1):23-8.

15. Fiala J, Brázdová Z. A comparison between the lifestyles of men and women-parents of school age children. Cent Eur J Public Health. 2000 May;8(2):94-100.

16. Gaesser GA. Exercise for prevention and treatment of cardiovascular disease, type 2 diabetes, and metabolic syndrome. Curr Diab Rep. 2007 Feb;7(1):14-9.

17. Slavíček J, Kittnar O, Fraser GE, Medová E, Konečná J, Žižka R, et al. Lifestyle decreases risk factors for cardiovascular diseases. Cent Eur J Public Health. 2008 Dec;16(4):161-4.
18. Chrysohoou C, Pitsavos C, Kokkinos P, Panagiotakos DB, Singh SN, Stefanadis C. The role of physical activity in the prevention of stroke. Cent Eur J Public Health. 2005 Sep;13(3):132-6.

19. Fiala J, Brázdová Z. "Know and improve your health": symbiosis of preventive project and preventive medicine education at the medical faculty in Brno. Cent Eur J Public Health. 1996 Dec;4(4):257-62.

20. US Department of Health \& Human Services [Internet]. Washington, D.C.: U.S. Department of Health \& Human Services [cited 2012 Mar 15]. 2008 Physical activity guidelines for Americans. Available from: http://www.health.gov/paguidelines/.

21. Tremblay MS, Warburton DER, Janssen I, Paterson DH, Latimer AE, Rhodes RE, et al. New Canadian physical activity guidelines. Appl Physiol Nutr Metab. 2011 Feb;36(1):36-46; 47-58.

22. Bateman LA, Slentz CA, Willis LH, Shields AT, Piner LW, Bales CW, et al. Comparison of aerobic versus resistance exercise training effects on metabolic syndrome (from the Studies of a Targeted Risk Reduction Intervention Through Defined Exercise - STRRIDE-AT/RT). Am J Cardiol. 2011 Sep 15;108(6):838-44.

23. Slentz CA, Bateman LA, Willis LH, Shields AT, Tanner CJ, Piner LW, et al. Effects of aerobic vs. resistance training on visceral and liver fat stores, liver enzymes, and insulin resistance by HOMA in overweight adults from STRRIDE AT/RT. Am J Physiol Endocrinol Metab. 2011 Nov;301(5):E1033-9.

24. Stensvold D, Tjønna AE, Skaug EA, Aspenes S, Stølen T, Wisløff U, et al. Strength training versus aerobic interval training to modify risk factors of metabolic syndrome. J Appl Physiol. 2010 Apr;108(4):804-10.

25. Katzmarzyk PT, Leon AS, Wilmore JH, Skinner JS, Rao DC, Rankinen T, et al. Targeting the metabolic syndrome with exercise: evidence from the HERITAGE Family Study. Med Sci Sports Exerc. 2003 Oct;35(10):17039.

26. Tuomilehto J, Lindström J, Eriksson JG, Valle TT, Hämäläinen H, IlanneParikka P, et al. Prevention of type 2 diabetes mellitus by changes in lifestyle among subjects with impaired glucose tolerance. N Engl J Med. 2001 May 3;344(18):1343-50

27. Knowler WC, Barrett-Connor E, Fowler SE, Hamman RF, Lachin JM, Walker EA, et al. Reduction in the incidence of type 2 diabetes with lifestyle intervention or metformin. N Engl J Med. 2002 Feb 7;346(6):393403.

28. Pan XR, Li GW, Hu YH, Wang JX, Yang WY, An ZX, et al. Effects of diet and exercise in preventing NIDDM in people with impaired glucose tolerance. The Da Qing IGT and Diabetes Study. Diabetes Care. 1997 Apr;20(4):537-44.

29. Shaw K, Gennat H, O'Rourke P, Del Mar C. Exercise for overweight or obesity. Cochrane Database Syst Rev. 2006 Oct 18;(4):CD003817.

30. Payne WR, Walsh KJ, Harvey JT, Livy MF, McKenzie KJ, Donaldson A, et al. Effect of a low-resource-intensive lifestyle modification program incorporating gymnasium-based and home-based resistance training on type 2 diabetes risk in Australian adults. Diabetes Care. 2008 Dec;31(12):2244-50.

31. Absetz P, Valve R, Oldenburg B, Heinonen H, Nissinen A, Fogelholm M, et al. Type 2 diabetes prevention in the "real world": one-year results of the GOAL Implementation Trial. Diabetes Care. 2007 Oct;30(10):246570 .

32. Laatikainen T, Dunbar JA, Chapman A, Kilkkinen A, Vartiainen E, Heistaro S, et al. Prevention of type 2 diabetes by lifestyle intervention in an Australian primary health care setting: Greater Green Triangle (GGT) Diabetes Prevention Project. BMC Public Health. 2007 Sep 19;7:249.

33. Ackermann RT, Finch EA, Brizendine E, Zhou H, Marrero DG. Translating the Diabetes Prevention Program into the community. The DEPLOY Pilot Study. Am J Prev Med. 2008 Oct;35(4):357-63.

34. Lipscomb ER, Finch EA, Brizendine E, Saha CK, Hays LM, Ackermann RT. Reduced 10-year risk of coronary heart disease in patients who participated in a community-based diabetes prevention program: the DEPLOY pilot study. Diabetes Care. 2009 Mar;32(3):394-6.

35. Noonan V, Dean E. Submaximal exercise testing: clinical application and interpretation. Phys Ther. 2000 Aug;80(8):782-807.

36. Foster C, Jackson AS, Pollock ML, Taylor MM, Hare J, Sennett SM, et al. Generalized equations for predicting functional capacity from treadmill performance. Am Heart J. 1984 Jun;107(6):1229-34.

37. Libardi CA, De Souza GV, Cavaglieri CR, Madruga VA, Chacon-Mikahil MP. Effect of resistance, endurance, and concurrent training on TNFalpha, IL-6, and CRP. Med Sci Sports Exerc. 2012 Jan;44(1):50-6.

38. Perri MG, Martin AD, Leermakers EA, Sears SF, Notelovitz M. Effects of group- versus home-based exercise in the treatment of obesity. J Consult Clin Psychol. 1997 Apr;65(2):278-85. 
39. Lakka TA, Laaksonen DE, Lakka HM, Männikkö N, Niskanen LK, Rauramaa $\mathrm{R}$, et al. Sedentary lifestyle, poor cardiorespiratory fitness, and the metabolic syndrome. Med Sci Sports Exerc. 2003 Aug;35(8):1279-86.

40. Katzmarzyk PT, Church TS, Blair SN. Cardiorespiratory fitness attenuates the effects of the metabolic syndrome on all-cause and cardiovascular disease mortality in men. Arch Intern Med. 2004 May 24;164(10):1092-7.

41. Lee DC, Sui X, Church TS, Lee IM, Blair SN. Associations of cardiorespiratory fitness and obesity with risks of impaired fasting glucose and type 2 diabetes in men. Diabetes Care. 2009 Feb;32(2):257-62.

42. King AC, Haskell WL, Taylor CB, Kraemer HC, DeBusk RF. Groupvs home-based exercise training in healthy older men and women. A community-based clinical trial. JAMA. 1991 Sep 18;266(11):1535-42.

43. Tambalis K, Panagiotakos DB, Kavouras SA, Sidossis LS. Responses of blood lipids to aerobic, resistance, and combined aerobic with resistance exercise training: a systematic review of current evidence. Angiology. 2009 Oct-Nov;60(5):614-32.

44. Balkau B, Mhamdi L, Oppert JM, Nolan J, Golay A, Porcellati F, et al. Physical activity and insulin sensitivity: the RISC study. Diabetes. 2008 Oct;57(10):2613-8

45. Østergård T, Andersen JL, Nyholm B, Lund S, Nair KS, Saltin B, et al. Impact of exercise training on insulin sensitivity, physical fitness, and muscle oxidative capacity in first-degree relatives of type 2 diabetic patients. Am J Physiol Endocrinol Metab. 2006 May;290(5):E998-1005.
46. Holloszy JO. Exercise-induced increase in muscle insulin sensitivity. J Appl Physiol. 2005 Jul;99(1):338-43.

47. Mora S, Cook N, Buring JE, Ridker PM, Lee IM. Physical activity and reduced risk of cardiovascular events: potential mediating mechanisms. Circulation. 2007 Nov 6;116(19):2110-8.

48. Autenrieth C, Schneider A, Döring A, Meisinger C, Herder C, Koenig $\mathrm{W}$, et al. Association between different domains of physical activity and markers of inflammation. Med Sci Sports Exerc. 2009 Sep;41(9):1706-13.

49. Verdaet D, Dendale P, De Bacquer D, Delanghe J, Block P, De Backer G. Association between leisure time physical activity and markers of chronic inflammation related to coronary heart disease. Atherosclerosis. 2004 Oct;176(2):303-10.

50. Cornelissen VA, Fagard RH, Coeckelberghs E, Vanhees L. Impact of resistance training on blood pressure and other cardiovascular risk factors: a meta-analysis of randomized, controlled trials. Hypertension. 2011 Nov;58(5):950-8

51. Cornelissen VA, Fagard RH. Effects of endurance training on blood pressure, blood pressure-regulating mechanisms, and cardiovascular risk factors. Hypertension. 2005 Oct;46(4):667-75.

Received June 4, 2012 Accepted in revised form November 24, 2012 